\begin{tabular}{c} 
International Journal of Engineering \&Technology, $7(1.8)(2018) 168-171$ \\
International Journal of Engineering \& Technology \\
Website: www.sciencepubco.com/index.php/IJET \\
Research paper \\
\hline
\end{tabular}

\title{
Detection of fault and current approximation by wavelet transform
}

\author{
D Narasimha rao ${ }^{1 *}$, Akhila Polocherla ${ }^{2}$, Madhuri Arumalla ${ }^{3}$ \\ ${ }^{1}$ Department of EEE KLEF Andhra Pradesh, India \\ ${ }^{2}$ Department of EEE KLEF Andhra Pradesh, India \\ ${ }^{3}$ Department of EEE KLEF Andhra Pradesh, India \\ *Corresponding author E-mail:narasimharao@kluniversity.in
}

\begin{abstract}
This paper acquaints a strategy with perceive the region of the normal fault on the power line for fast and strong activity of confirmation designs. The model is made in MATLAB to deliver the important segment of the transient parameters at a similar period. Each cycle of waveform, covers before blame and after blame information is inaccessible for examination. The DWT is used for data pre-preparing. MATLAB writing computer programs is used to replicate uncommon. Working and blame conditions on high voltage transmission line, to be particular LG blame, LL blame, LLG and LLLG cut off.
\end{abstract}

Keywords: Discrete Wavelet Change (DWT), Transients, MATLAB.

\section{Introduction}

Blame area and separation is the major issue in control outline building in request for the fault to clear rapidly and re-establish control supply at the initial chance with least interfering. This is fundamental for dependable operation of energy gear and fulfilment of client. Before a few methods were connected for assessing shortcoming area with various methods, for example, line impedance based numerical techniques, travelling wave techniques and Fourier examination. These days, peak recurrence segments rather than customary strategy have been utilized. Fourier change were utilized to unique crucial recurrence segments yet it has been demonstrated that Fourier Change based investigation now and then don't perform time limitation of time shifting signs with adequate precision. As of late wavelet change has been utilized widely for assessing issue area precisely. The most commanding normal for wavelet change is to divide the wave on time scale as opposed to in repetition area. Subsequently a DWT is utilized as a part of this paper since it is extremely successful in distinguishing deficiency created motions as time difference .This articleuses a wavelet change based blame locator calculation. For this reason, $735 \mathrm{KV}, 300 \mathrm{~km}, 50 \mathrm{~Hz}$ power line is mimicked utilizing power framework Block set of MATLAB.

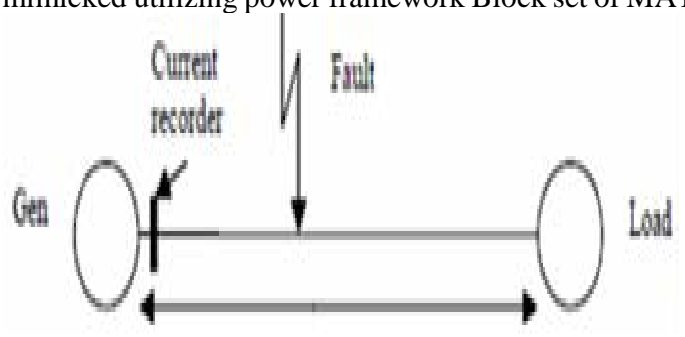

Fig 1: Block diagram of the proposed system
Wavelet change (WT) is a technical system aimed at some utilization of flag preparing. Wavelet is substantially more effective than regular technique in handling the stochastic flag due to investigating the wave with respect to time scale region. In wavelet change the band of investigation can be balanced with the goal that little reappearance and peak reappearance parts can be viewed by distinctive factors. As of late WT is generally utilized as a part of flag handling application, for example, de noising, separating, and picture pressure. Numerous example acknowledgment calculations were created in view of the wavelet transform. As indicated by scale factors utilized the wavelet can be sorted into various areas.

The basic thought of wavelet change is that the change ought to permit just changes in time augmentation, yet not shape. This is influenced by picking appropriate premise works that take into account this. Changes in the time augmentation are relied upon to affirm to the comparing investigation recurrence of the premise work. Wavelet change is a scientific strategy utilized for some uses of flag handling. The wavelet change is in certainty an unending arrangement of different change contingent upon the unit work utilized for its calculation.

It is significantly more effective than tradition technique in handling the flag as a result of examining the waveform in time scale area in wavelet change the band of examination can be balanced with the end goal that little recurrence and great recurrence segments can be separated by various scale factors. Windowed Fourier change likewise in part accomplishes this objective however with the constraint of utilizing a settled width windowing capacity.

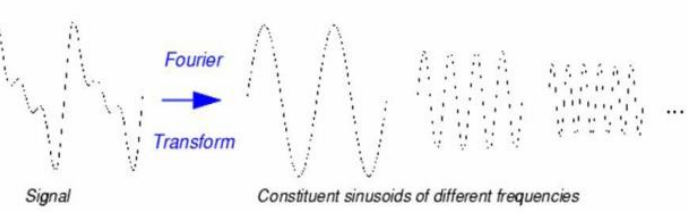

\section{Wavelet Transform}




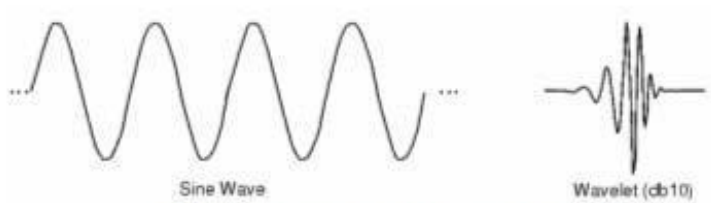

The wavelet transform

Fig 2: Wavelet Transform

In this work, the DWT was utilized. For any function (f),DWT is composed as:

$$
\operatorname{DWT}(m, k)=\frac{1}{\operatorname{sqrt}\left(a_{0}^{m}\right)} \sum_{n} x(n) g\left(\frac{k-n b_{0} a_{0}^{m}}{a_{0}^{m}}\right)
$$

$\mathrm{g}(\mathrm{n})$ is the mother wavelet,

$\mathrm{x}(\mathrm{n})$ is the information flag, and

$\mathrm{k}$ is whole number variable alludes to a specific example number in an information flag.

DWT is actualized by utilizing a multi-arrange filter.

\section{Why do we use wavelet transform instead of relays?}

We use wavelet transform instead of relays or any other available, because of the fact that wavelet transform provide us with the reliable performance which has the ability to detect the fault within less time when compared to the performance of relays. Since the wavelet differentiate various frequencies and can operate within less time.

\section{Transmission Line}

An over hear transmission conducting line is unique of the fundamental segments in each electrical system. Power lines associate the producing plants and load centres. As the producing stations are far away from the load focuses they keep running more than many kilometres. The faults can weaken the power systemso they should be solved quickly. Faultcorrection is vital issue in control framework building all together that to clear faults rapidly and re-establishes control supply as quickly as time permits with least intrusion. At the point when fault happens on an power line, it is essential to recognize it and to discover its area keeping in mind the end goal to make important repairs and to re-establish control when conceivable. In this manner, an exact fault area on the line is a critical prerequisite for a changeless fault. Transmission conducting line security is an imperative issue in control framework designing in light of the fact that $85-87 \%$ of energy framework issues are happening in transmission conducting lines.

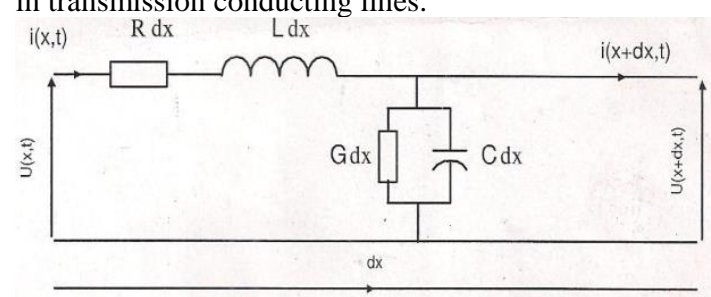

Fig 3: Line model of a single transmission conducting line system

\section{Basic Ideas \& Proposed Algorithm}

Issues occur at various stages of transmission conducting line. All the data of the information is gathered from supply end of the system. Blame signs (voltages and streams) are assembled for acycle of $50 \mathrm{~Hz}$. Discrete frame signal consideration is done, tests of current what's more, examples of voltage are taken for a wide range of deficiencies. The wavelet change is displayed, MATLAB based reenactment for evaluating deficiency area on transmission conducting lines. The re-enactment is created as a single end recurrence centred strategy what's more, utilized both voltage and current impact coming about from remote end of the power framework. The pre-fault and post-fault data is covered in one cycle which is preoccupied for examination. The DWT is utilized for information preprocessing. Discrete Wavelet changeis connected for deciding the key part, which can be helpful to give significant data to the Separation hand-off to react to a fault. MATLAB programming is utilized to reproduce diverse working and blame conditions on ultrapower high voltage transmission conducting line, to be specific LG fault, LL fault, LLG fault and also, current zero in all the phases.

\section{Simulation Block Diagram}

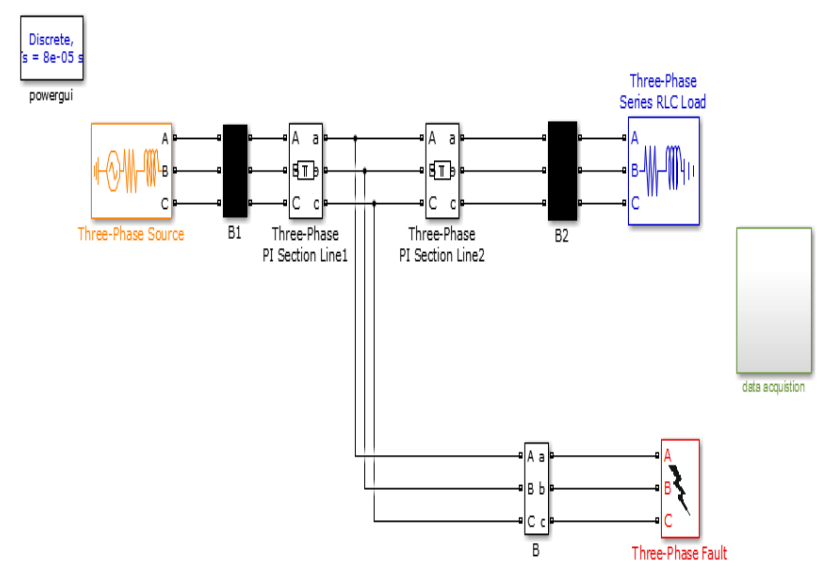

Fig 3: MATLAB Simulation Block diagram

Table 1: Line Data

\begin{tabular}{|l|l|}
\hline $\mathrm{L}(\mathrm{H} / \mathrm{Km})$ & $0.9337 * 10^{-3}$ \\
\hline $\mathrm{C}(\mathrm{F} / \mathrm{Km})$ & $12.74 * 10^{-9}$ \\
\hline Length & $190 \mathrm{Km}$ \\
\hline $\mathrm{V}=\frac{1}{\sqrt{L . C}}$ & $289 \mathrm{Kv}$ \\
\hline
\end{tabular}

Table 2: Source Data

\begin{tabular}{|l|l|}
\hline Supply voltage & $132 \mathrm{kv}$ \\
\hline Frequency & $50 \mathrm{~Hz}$ \\
\hline Fault Time & $0.33 \mathrm{sec}$ \\
\hline Sampling Time & $8 * 10^{-8}$ \\
\hline
\end{tabular}

\section{Simulation Graphs:}

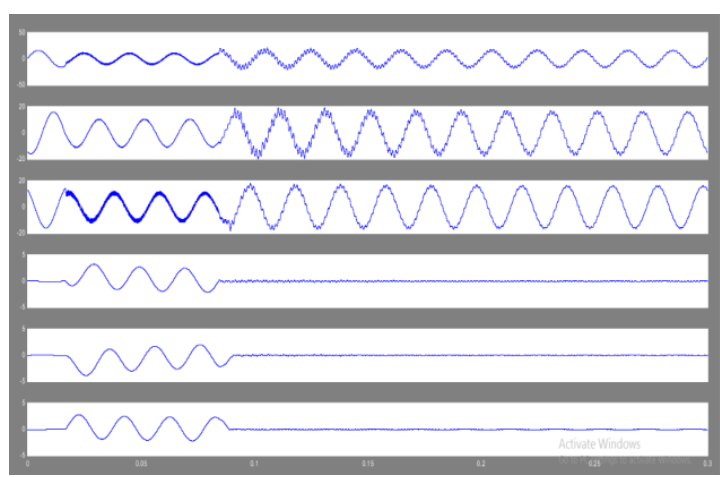

Fig 4: Voltage and current waveforms of Bus-1 


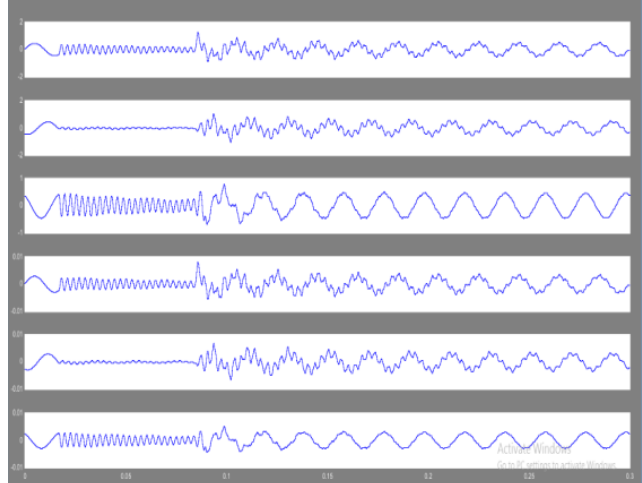

Fig 5: Voltage and current waveforms of Bus-2

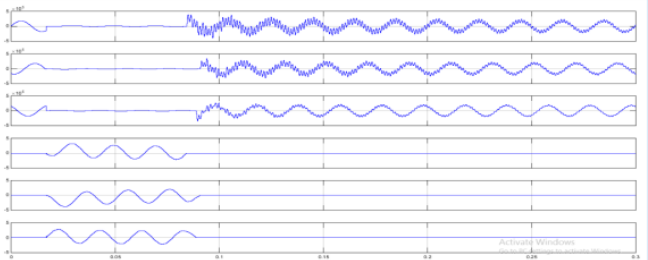

Fig 6: Voltage and currents of fault Bus

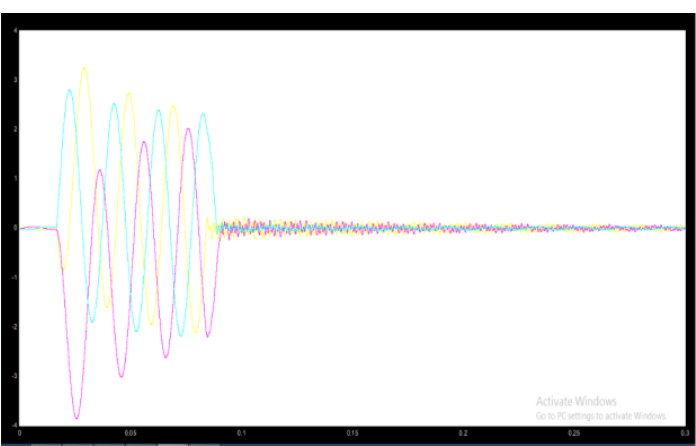

Fig7: Current at Bus-1

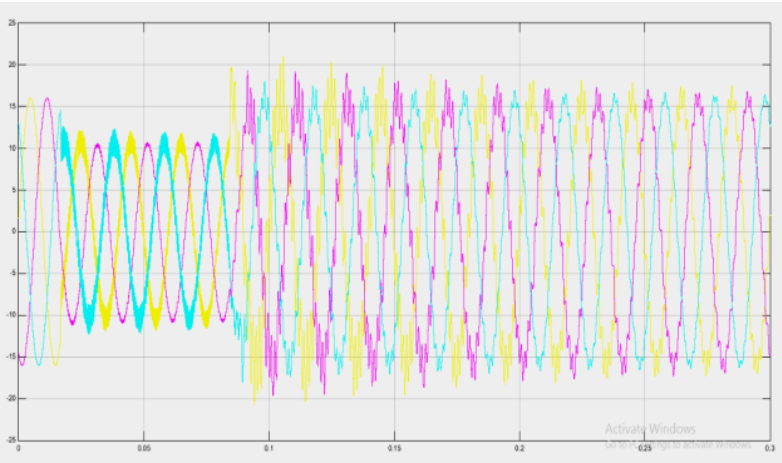

Fig 8: Voltage at Bus-1

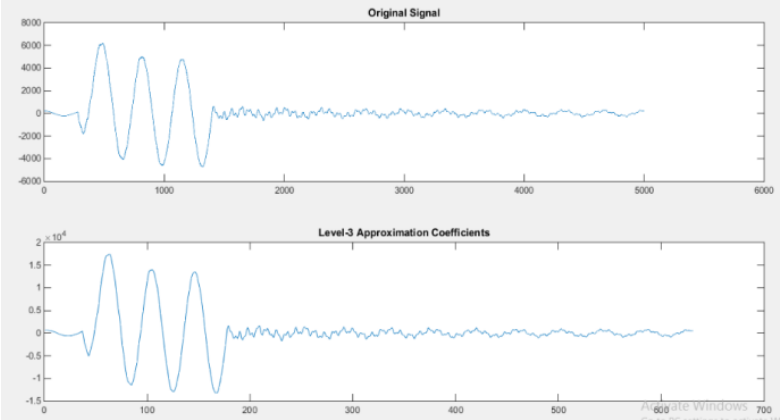

Fig 9: Current at Phase- A after using Wavelet change(LG-Fault)

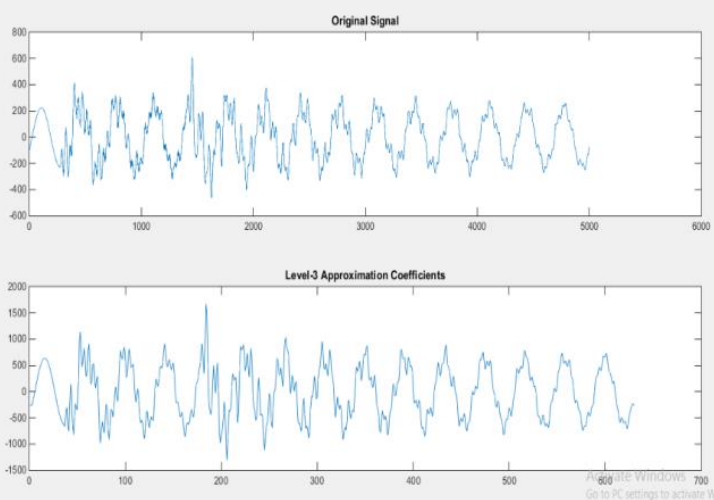

Fig 10: Current at Phase- B after Using wavelet change

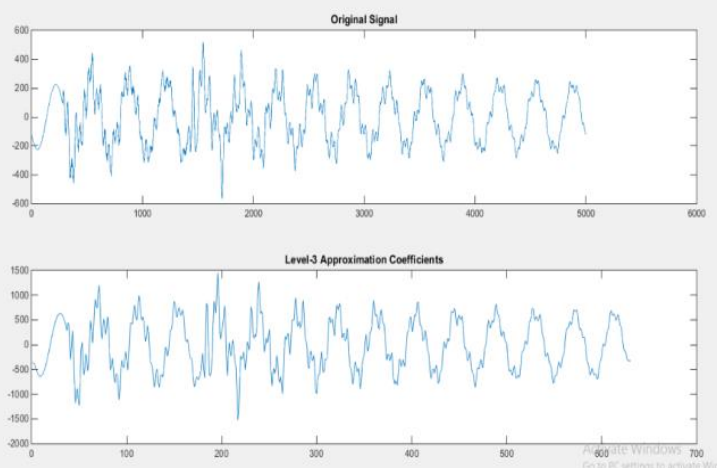

Fig 11: Current at Phase-C after using wavelet transforms

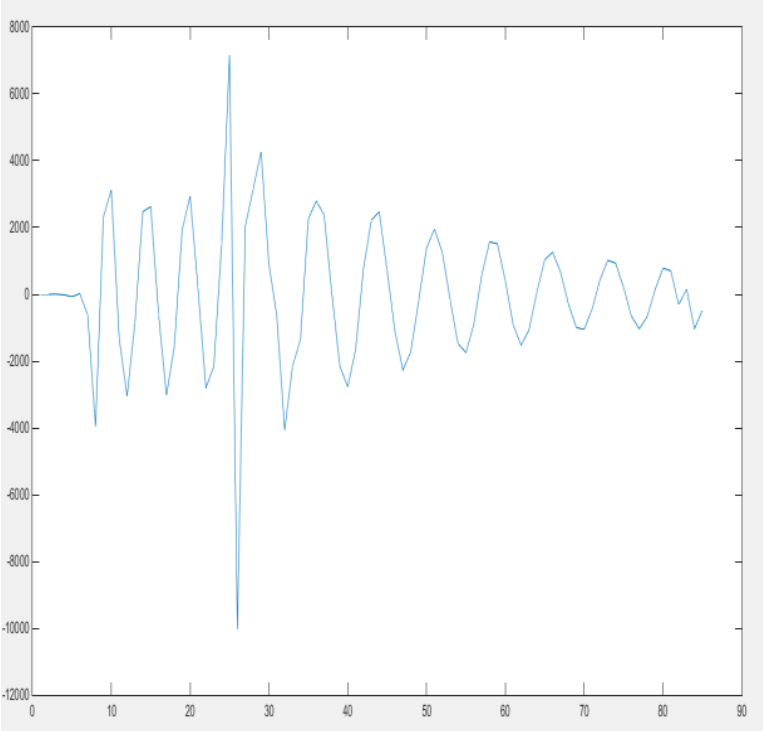

Fig 12: dB 4- waveform representation

\section{Results}

This paper presents a new idea of how wavelet transform can be used in order to detect the blame in the transmission conducting system. This detection method requires a MATLAB Simulink software system in which wavelet toolbox is the tool used to detect the blame in the system. Through this tool we can also detect the approximation current which gives the approximate current in the system either during the blame, post-blame or before the occurrence of blame. Prior to the approximated current we can also get the detail co-efficient of current at various levels.

\section{Conclusion}

Usage of the wavelet transformationtend to evaluate the blame area on transmission conducting line has been observed. The capacity of 
wavelets is to decay the flag into recurrence groups in both time and recurrence permits precise blame detection. The waveforms attained from SIMULINK have been changed over as a MATLAB document for highlight extraction. DWT is being utilized to examine the flag to get the quantities for assessing the blame area. At last it was demonstrated that the proposed technique is sufficiently exact to be utilized as a part of discovery of transmission line blame area.

\section{References}

[1] A.H.Osman and O.P.Malik," Transmission line protection based on wavelet transform" ,IEEE Trans. Power Delivery,Vol.19,No.2,pp.515-523,April 2004.

[2] Sukumar M.Brahma "Fault location on a transmission line using Synchronized voltage measurements", IEEE Transactions on power Delivery,Vol.19,No.4,pp.1619-1622,2004

[3] Feng Liang and B.JeyaSurya,'Transmission line protection using wavelet transform based algorithm", IEEE Trans. Power Delivery, Vol.19,No.2,pp.545-553,April 2004.

[4] Ching-shan Chen, Ying-Hong Lin, Chih-Wen Lin "A new PMU based fault detection/loction technique for transmission lines with consideration of arcing fault discrimination-Part I\&\&II", IEEE Transactions on power Delivery,Vol.19,No.4,pp.1587-1601,2004

[5] Robi Polikar," The wavelet Tutorial part-2 for Fourier Transform \& Short term Fourier Transform and part-3 for continuous Wavelet transform", Ames,Iowa, 2nd Edition,1996

[6] B. Das and J. V. Reddy, "Fuzzy-logic-based fault classification scheme for digital distance protection," IEEE Transactions on Power Delivery, Vol. 20, pp. 609-616, April 2005.

[7] P. K. Dash, S. R. Samantaray and G. Panda, "Fault classification and section identification of an advanced series-compensated transmission line using support vector machine," IEEE Transactions on Power Delivery, Vol. 22, No. 1, pp. 67-73, January 2007. 